\title{
Mechanics of multidimensional isolated horizons
}

\author{
Mikołaj Korzyński ${ }^{1}$, Jerzy Lewandowski ${ }^{1,2,3}$ and Tomasz Pawłowski ${ }^{1,2}$ \\ ${ }^{1}$ Instytut Fizyki Teoretycznej, Uniwersytet Warszawski, ul. Hoża 69, 00-681 Warsaw, Poland \\ 2 Center for Gravitational Physics and Geometry, Physics Department, 104 Davey, Penn State, \\ University Park, PA 16802, USA \\ ${ }^{3}$ Max-Planck-Institut für Gravitationsphysik, Albert-Einstein-Institut, 14476 Golm, Germany
}

Received 2 February 2005, in final form 21 March 2005

Published 10 May 2005

Online at stacks.iop.org/CQG/22/2001

\begin{abstract}
Recently, a multidimensional generalization of the isolated horizon framework has been proposed (Lewandowski and Pawłowski 2005 Class. Quantum Grav. 22 1573-98). Therein the geometric description was easily generalized to higher dimensions and the structure of the constraints induced by the Einstein equations was analysed. In particular, the geometric version of the zeroth law of black-hole thermodynamics was proved. In this work, we show how the IH mechanics can be formulated in a dimension-independent fashion and derive the first law of $\mathrm{BH}$ thermodynamics for arbitrarily dimensional $\mathrm{IH}$. We also propose a definition of energy for non-rotating horizons.
\end{abstract}

PACS numbers: $04.70 . \mathrm{Bw}, 04.70 . \mathrm{Dy}, 04.50 .+\mathrm{h}$

\section{Introduction}

Four-dimensional isolated horizons (IH) proved to be a useful tool for studying black-hole mechanics, thermodynamics and even quantum theory $[15,16]$. Being quasi-local they are also useful for numerical relativity (gravitational wave investigation, black-hole merger studies). It is, therefore, interesting to investigate whether the notion of IH exists in higher dimensions and check if their physical properties are similar.

The Hamiltonian formulation of general relativity combined with the IH framework made it possible to formulate the first law of black-hole thermodynamics for both rotating and non-rotating black holes $[2,5,17]$. Consider spacetimes with axially symmetric IHs (the spacetimes themselves do not have to be symmetric at all). The first law arises naturally as one investigates the transformations of the phase space given by flows of vector fields (time translation). One may ask about the conditions for such transformations to be generated by a Hamiltonian. It turns out (see [2]) that the only condition constrains the value of the vector field on the horizon. The condition implies that the generating Hamiltonian must be a function of the horizon area and angular momentum solely-but does not constrain this function in any way. One can, however, fix the Hamiltonian function (and therefore the IH energy value) 
by requiring that it agrees with the ADM mass in the case of stationary, asymptotically flat solution. Note that due to the Kerr solution uniqueness theorems such fixing is consistent: if the solution is stationary and asymptotically flat, it must be a Kerr metric and the ADM mass must depend on $A$ and $J$ in the Kerr-like manner.

This work deals with a $(N+2)$-dimensional $(N>2)$ generalization of rotating isolated horizons [1]. We first check that the Hamiltonian formalism leads to the same conditions on the Hamiltonian function as in the 4D case. This result is valid for any rotating (axially symmetric) weakly isolated horizon in any dimension. In order to define the energy however, we restrict ourselves to non-rotating ones. This is due to the fact that the general uniqueness theorem for the axially symmetric spacetimes fails in higher dimensions [10]. One cannot assign safely the energy function using some family of solutions analogous to Kerr solutions in 4D since there exist other families with different ADM mass for given horizon area and angular momentum $[9,10]$. One would have to argue somehow why the choice of one family of solutions is more physical than another.

It is true, however, that the conditions for the topology of the horizon to be a sphere and the existence of two axes of symmetry are strong enough to prove the uniqueness of solution in five dimensions [11].

Nevertheless, only in the static, non-rotating case do there exist general uniqueness theorems for arbitrary dimension and for $\sigma$-model, vacuum and charged black holes [6-8]. Since we deal with the vacuum case, we will assume the energy dependence on the horizon area as in the generalized Schwarzschild case.

\section{Weakly isolated horizons}

In this section we recall the definition of non-expanding horizons (NEH) [1], spell out the definition of weakly isolated horizons (WIH) and discuss those of their properties which will prove relevant in the next sections.

The following convention of indices will be adopted:

- Greek indices will be used for objects defined on the whole $(N+2)$-dimensional tangent space of $M$,

- small Latin letters for objects defined on or contained in the $(N+1)$-dimensional subspace (tangent to the horizon) and

- capital Latin letters for the $N$-dimensional subspace (tangent to a cut).

The symbol ' $d$ ' will stand for the exterior derivative for any manifold. The abstract index notation will be used whenever convenient.

\subsection{Non-expanding horizons}

Let $\Delta$ be an $(N+1)$-dimensional null surface in an $(N+2)$-dimensional spacetime $M$ equipped with a metric tensor field of the signature $(-,+, \ldots,+)$ which satisfies Einstein equations with or without cosmological constant ${ }^{4}$, and let $l$ denote a non-vanishing normal vector. If the expansion of $l$ vanishes everywhere on $\Delta$, then this property is independent of the choice of $l$ and we call $\Delta$ a non-expanding null surface. This assumption, combined with a mild energy condition $T_{\alpha \beta} l^{\alpha} l^{\beta} \geqslant 0$, implies a restriction [1] on the Ricci tensor:

$$
R_{\alpha \beta} l^{\alpha} l^{\beta}=0 \text {. }
$$

4 In the main part concerning the first law we will restrict ourselves to the vacuum case without cosmological constant. 
It also implies that the metric tensor $q$ induced on $\Delta$ (degenerate) metric is Lie dragged by any null vector field tangent to $\Delta$

$$
\mathcal{L}_{l} q=0 .
$$

It follows that there exists on $\Delta$ a differential 1-form $\omega(l)$ called the rotation potential, such that at every $x \in \Delta$ and for every $X \in T_{x} \Delta$,

$$
X^{c} \nabla_{c} l=X^{c} \omega_{c}^{(l)} l
$$

where $\nabla$ is the covariant derivative. Moreover, a non-expanding surface $\Delta$ is called a nonexpanding horizon $(\mathrm{NEH})$ if there exists an embedding

$$
\hat{\Delta} \times[0,1] \mapsto M
$$

where $[0,1] \subset \mathbb{R}$ stands for the interval, such that:

(1) $\Delta$ is the image,

(2) $\hat{\Delta}$ is an $N$-dimensional compact, connected manifold,

(3) for every maximal null curve in $\Delta$ there exists $\hat{x} \in \hat{\Delta}$ such that the curve is the image of $\{\hat{x}\} \times[0,1]$.

It follows that the manifold $\hat{\Delta}$ is the space of the null geodesics generating $\Delta$ and there is a natural projection

$$
\Pi: \Delta \rightarrow \hat{\Delta}
$$

Roughly speaking, the definition of a non-expanding horizon amounts to an extra condition on the topology of a non-expanding null surface ${ }^{5}$.

\subsection{The definition of weakly isolated horizons}

A weakly isolated horizon is a pair: a NEH $\Delta$ equipped with a class [ $l]$ of non-vanishing vector fields, normal to $\Delta$ (i.e. null and tangent) such that:

(i) for every $l, l^{\prime} \in[l]$ there is a positive constant $b$ such that

$$
l^{\prime}=b l,
$$

(ii) the rotation 1 -form potential $\omega^{(l)}$ is Lie dragged by $l$

$$
\mathcal{L}_{l} \omega^{(l)}=0 .
$$

The class $[l]$ will be often referred to as the WIH flow. Note that the rotation 1-form potential (3) is insensitive to the constant re-scalings (6). Indeed, for every function $b: \Delta \rightarrow \mathbb{R}$ and a null, nowhere vanishing vector field $l$ tangent to a non-expanding horizon, we have

$$
\omega^{(b l)}=\omega^{(l)}+\mathrm{d} \ln b .
$$

Therefore from now on we will skip the suffix $(l)$, whenever a WIH is given.

We will summarize several basic facts concerning NEHs and WIHs. For proofs and further explanations see [1] and also the four-dimensional case results [4, 5].

To begin with, note that the integral curves of a vector field $l$ normal to NEH $\Delta$ are null and geodesic in the sense that

$$
l^{\beta} \nabla_{\beta} l=\kappa^{(l)} l .
$$

\footnotetext{
5 Note that no geodesic completeness assumption has been made. Therefore a NEH is not assumed to be extending to past or future infinity. Physically, it means that a NEH can be formed some time in the past as well as destroyed or distorted some time in the future.
} 
The function coefficient $\kappa^{(l)}$ is called the surface gravity. Under a bit stronger energy condition that $T_{\alpha \beta} l^{\beta}$ is causal and future-pointing one may also prove the zeroth law of black-hole thermodynamics which states that

$$
\mathrm{d} \kappa^{(l)}=\mathcal{L}_{l} \omega^{(l)}
$$

Using the zeroth law it is easy to prove that every non-expanding horizon $\Delta$ admits a large class of null vector fields $l$, each of which defines a distinct weakly isolated horizon [1].

In particular, it follows that the surface gravity is necessarily constant for every WIH. If $\kappa^{(l)}=0$, the WIH is called extremal. Given a WIH there exists a freedom of rescaling the vector $l^{\alpha}$ by a constant, positive factor accompanied by the same rescaling of $\kappa^{(l)}$. Therefore in the extremal WIH case $\kappa^{(l)}$ is determined as 0 . Otherwise, in the non-extremal case, its exact value depends on the choice of $l^{\alpha} \in[l]$, while its sign is determined.

\subsection{Good cuts foliation}

Let $(\Delta,[l])$ be a non-extremal weakly isolated horizon. There exists a natural foliation [4], [1] of $\Delta$ distinguished by the geometry of $(\Delta,[l])$. It is defined as follows: let $\tilde{\Delta} \subset \Delta$ be any leaf of the foliation. Then, the pullback $\tilde{\omega}_{\alpha}$ of the rotation 1 -form potential onto $\tilde{\Delta}$ is divergence free,

$$
\tilde{q}^{A B} \tilde{D}_{A} \tilde{\omega}_{B}=0
$$

where $\tilde{q}$ is the metric tensor induced on $\tilde{\Delta}$ and $\tilde{D}$ is the corresponding torsion-free covariant derivative. If we assume that the leaves are global cross-sections of the maximal analytic extension of $\Delta$, then the foliation formed by them is unique [1,4]. It is called a good cuts foliation of a WIH $(\Delta,[l])$.

\subsection{Symmetries and symmetric WIHs}

We consider non-extremal WIHs $(\Delta,[l])$ here, of induced metric tensor $q$ and the rotation 1 -form potential $\omega$.

A vector field $X$ tangent to a WIH $(\Delta,[l])$ is called a symmetry generator whenever it is true that

$$
\mathcal{L}_{X} l=a l, \quad \mathcal{L}_{X} q=0, \quad \mathcal{L}_{X} \omega=0 .
$$

For example, a vector field of the form $f l$, where $f$ is a function and $l \in[l]$, is an asymmetry generator if and only if $f=$ const. Indeed,

$$
\mathcal{L}_{f l} \omega=f \mathcal{L}_{f} \omega+\kappa^{(l)} \mathrm{d} f=\kappa^{(l)} \mathrm{d} f .
$$

Hence, every WIH admits the null symmetry generators $l \in[l]$.

Suppose now a given non-extremal WIH $(\Delta,[l])$ admits another symmetry generator, a vector field $X$ which is not everywhere null on $\Delta$. It follows from the first equality in (12) that the projection $\Pi_{*} X$ is a well-defined vector field on the base manifold $\hat{\Delta}$. Due to the second equality in (12) we conclude that

$$
\mathcal{L}_{\Pi_{*} X} \hat{q}=0,
$$

hence the vector field $\Pi_{*} X$ is a Killing vector of the metric tensor defined on the base manifold $\hat{\Delta}$. This shows that a generic WIH does not admit non-null symmetries. We now use the good cuts foliation of $\Delta$ to lift $\Pi_{*} X$ to a vector field $\tilde{X}^{a}$ defined on $\Delta$, tangent to the good cuts at every point, and such that

$$
\Pi_{*} \tilde{X}=\Pi_{*} X .
$$


Therefore,

$$
X=f l+\tilde{X},
$$

where the factor $f$ is a function defined on $\Delta$.

We claim that again

$$
\mathrm{d} f=0 \text {. }
$$

To see this, note first that a stronger condition than the first equation in (12) is necessarily true, namely

$$
\mathcal{L}_{X} l=0
$$

Indeed,

$$
0=\mathcal{L}_{X} \kappa=\mathcal{L}_{X}\left(l^{a} \omega_{a}\right)=a \kappa^{(l)},
$$

due to the last equality in (12). Secondly, let us exercise the invariant character of the good cuts foliation. Introduce a function $v: \Delta \rightarrow \mathbb{R}$ constant on each leaf of the good cut foliation, and such that $l^{a} D_{a} v=1$. The derivative $\mathrm{d} v$ is uniquely defined by $l$ and the foliation, and both are preserved by the local flow of $X$, therefore the derivative is necessarily preserved by the flow of $X$. Hence

$$
0=\mathcal{L}_{X} \mathrm{~d} v=\mathrm{d} f .
$$

Finally, it follows that the vector field

$$
\tilde{X}=X-f l
$$

generates a WIH symmetry itself.

Concluding, if a WIH $(\Delta,[l])$ admits a non-null vector field $X$ generating a symmetry, then it admits another symmetry generator $\phi$ tangent to the leaves of the good cut foliation, and such that

$$
X=f l+\phi, \quad \text { where } \quad f=\text { const. }
$$

The vector field

$$
\hat{\phi}=\Pi_{*} \phi
$$

is a Killing vector field of the metric tensor $\hat{q}_{A B}$ induced in the space $\hat{\Delta}$ of the null geodesics in $\Delta$.

Basically, the argument presented above is the same as that of [5] (due to [1]), except a small gap in the proof of (17) which was filled in this section.

\section{The phase space of Einstein vacuums admitting WIH}

As in [5], throughout this paper we will use the covariant phase-space formalism, in a version admitting causal boundaries of the considered spacetime. An exhaustive description of this formalism can be found for example in [2] (see also [12, 17] for an alternative framework).

Our covariant phase space $\Gamma$ is, briefly speaking, the space of all the solutions of the vacuum Einstein equations (without cosmological constant) which admit a non-expanding horizon. We use the Einstein-Palatini formulation of gravity. The fields are defined on a given space $M$, a region of a spacetime $M^{\prime} . M$ is contained between three sub-manifolds: $\Delta$, $M_{0}$ and $M_{1}$, equipped by each point in the phase space with, respectively, a non-expanding horizon structure, and spacelike surface structures. Below, we first specify the assumptions about $M$, and secondly the boundary conditions. In section 8 we discuss the issue of gauge 


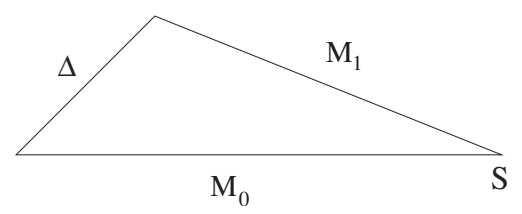

Figure 1. The quasi-local case: the spacetime region under consideration is compact and closed, bounded by three sub-manifolds with boundary $M_{0}, M_{1}$ and $\Delta ; M_{0}$ and $M_{1}$ are bounded by co-dimension 2 compact sub-manifolds, slices of $\Delta$, and by a single, co-dimension 2 , compact sub-manifold $S$.

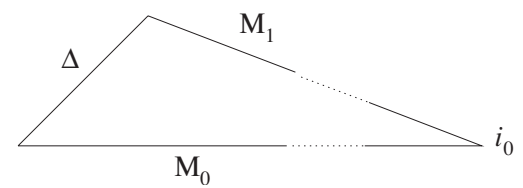

Figure 2. The asymptotically flat spacetime case: the spacetime region under consideration is infinite, bounded by sub-manifolds with boundary $M_{0}, M_{1}$ and $\Delta ; M_{0}$ and $M_{2}$ are bounded by co-dimension 2 compact sub-manifolds, slices of $\Delta ; i_{0}$ stands for the assumption that the gravitational fields be asymptotically flat.

dependence/invariance of our boundary conditions and the invariance of the resulting first law derived in section 7 .

$M$ is a closed region of a manifold $M^{\prime}$, and

$$
\dot{M}=M_{0} \cup \Delta \cup M_{1} \text {. }
$$

As in the previous sections, $\Delta=\hat{\Delta} \times[0,1]$ where $\hat{\Delta}$ is a compact, connected $N$-dimensional manifold. Our calculations will be valid for either of the following two cases:

- The properly quasi-local case, figure 1 . The space $M$ is compact. The surfaces $M_{0}$ and $M_{1}$ are compact, co-dimension 1 sub-manifolds with boundary, the boundary $\partial M_{0}$ (respectively, $\left.\partial M_{1}\right)$ consists of an intersection $\tilde{\Delta}_{0}\left(\tilde{\Delta}_{1}\right)$ with $\Delta$, and a co-dimension 2 compact sub-manifold $S$.

- The asymptotically flat case, figure 2. $M$ is an infinite region in $M^{\prime}$. The surfaces $M_{0}$ and $M_{1}$ are co-dimension 1 infinite sub-manifolds with boundary. The boundary $\partial M_{0}$ (respectively, $\left.\partial M_{1}\right)$ consists of an intersection $\tilde{\Delta}_{0}\left(\tilde{\Delta}_{1}\right)$ with $\Delta$.

In both cases we assume that the metric tensors under consideration extend smoothly to a neighbourhood of $M$ in $M^{\prime}$.

Additionally, in the second case we assume that the metric tensor fields are asymptotically flat ${ }^{6}$.

On the manifold $\Delta$ we fix the Cartesian product structure $\Delta=\hat{\Delta} \times[0,1]$ and on the manifold $\hat{\Delta}$ we fix an additional vector field $\hat{\phi}$ later used in the definition of the angular momentum ${ }^{7}$.

6 That is, we assume that the manifold $M$ minus some neighbourhood of $\Delta$ is diffeomorphic to $S_{N} \times \mathbb{R} \times[0,1]$, where $S_{N}$ is diffeomorphic to an $N$-dimensional sphere, and there exist spherical coordinates $\left(x^{\alpha}, r, t\right)$, flat with respect to some Minkowski metric tensor $g_{\mathrm{M} \mu \nu}$, such that for large $r$ the metric tensor components satisfy: $g_{\mu \nu}=g_{\mathrm{M} \mu \nu}+O\left(\frac{1}{r^{N-1}}\right)$, and $\partial_{\rho} g_{\mu \nu}=O\left(\frac{1}{r^{N}}\right)$.

7 In the four-dimensional case, when the horizon topology is $S^{2}$, it was proven that $\hat{\phi}$ must generate $S O(2)$ rotations, i.e. it must have closed orbits and its flow must be the identity map for some value of the flow parameter [5]. The normalization of the vector is later fixed when the resulting angular momentum formula is compared with Komar's. In our case we cannot exclude that our vector field can have more complicated orbits. 


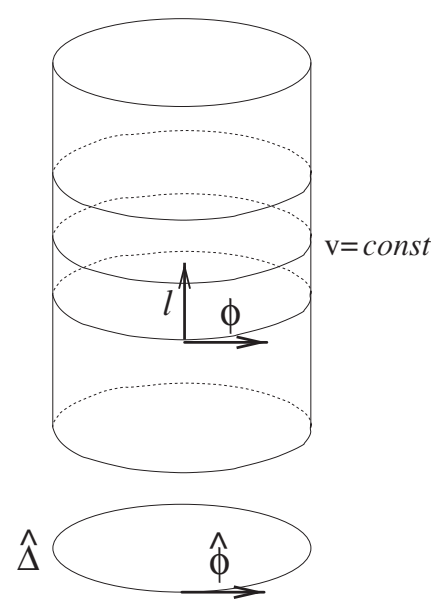

Figure 3. The structure fixed in $\Delta=\hat{\Delta} \times[0,1]$ : the Cartesian product structure, the natural coordinate $v$ on $[0,1]$ extended to $\Delta$, the vector field $\frac{\mathrm{d}}{\mathrm{d} v}$ extended to $\Delta$ and denoted by $l$, and a vector field $\phi$, the natural extension to $\Delta$ of a vector field $\hat{\phi}$ fixed on $\hat{\Delta}$.

Out of these data we also construct on $\Delta$ (see figure 3): (i) a vector field $l$, the natural extension to $\hat{\Delta} \times[0,1]$ of the vector field $\frac{\mathrm{d}}{\mathrm{d} v}, v:[0,1] \rightarrow[0,1]$ being the natural coordinate on the product, (ii) a function $v: \Delta \rightarrow \mathbb{R}$, the natural extension of the function $v$ defined on $[0,1]$, (iii) a vector field $\phi$, the natural extension of $\hat{\phi}$ to $\Delta=\hat{\Delta} \times[0,1]$.

On $M$ we consider vacuum gravitational fields such that $\Delta$ is a non-expanding horizon and $l$ defines a WIH on $\Delta$. Every gravitational field $g$ is determined by a co-frame, i.e. a sequence of differential 1-forms $\left(e^{1}, \ldots, e^{N+2}\right)$ normalized such that

$$
g=-e^{N+1} \otimes e^{N+2}-e^{N+2} \otimes e^{N+1}+\hat{\eta}_{A B} e^{A} \otimes e^{B},
$$

where $\hat{\eta}_{A B}, A, B=1, \ldots, N$, is the unit matrix $\operatorname{diag}(1, \ldots, 1)$. We assume that $M$ is oriented and the volume form $e^{1} \wedge \cdots \wedge e^{N+2}$ agrees with its orientation. We also assume the vector field $l$ defined on $\Delta$ to be future-pointing. We will use the Palatini framework, therefore we introduce an additional field, which is an anti-symmetric matrix $\left(\Gamma^{\alpha \beta}\right)^{\alpha, \beta=1, \ldots, N+2}$ of 1 -forms referred to as the connection 1-forms.

We formulate now the boundary conditions at $\Delta$ explicitly, in the technical way. Given a differential $n$-form $w$ in $M$, its pullback to $\Delta$ will be denoted by $w_{\Delta}$.

We assume every co-frame $\left(e^{1}, \ldots, e^{N+2}\right)$ considered in $M$ and about its dual vector frame $\left(e_{1}, \ldots, e_{N+2}\right)$ to satisfy

$$
\begin{aligned}
& \left.e_{N+1}\right|_{\Delta}=l \\
& \left(e^{N+1}\right)_{\Delta}=\mathrm{d} v \\
& \left(e^{N+2}\right)_{\Delta}=0 .
\end{aligned}
$$

The pullbacks to $\Delta$ of the connection 1-forms are subject to the following conditions:

$$
\begin{aligned}
& \left(\Gamma^{N+2}{ }_{1}\right)_{\Delta}=\cdots=\left(\Gamma^{N+2}{ }_{N}\right)_{\Delta}=0, \\
& \mathcal{L}_{l}\left(\Gamma^{N+1}{ }_{N+2}\right)_{\Delta}=0 .
\end{aligned}
$$

Conditions (26), (28) imply that $\Delta$ is null, condition (29) is equivalent to the assumption that $\Delta$ be a non-expanding horizon contained in a vacuum spacetime (it is exactly equivalent to (2)), whereas (30) (meaning the same as (7)) is the necessary and sufficient condition for the vector field $l$ to form a WIH together with $\Delta$. 
Just to simplify the calculations we introduce a short-hand notation for the following $N+2-k$-forms

$$
\Sigma_{\alpha \beta \ldots \gamma}=\frac{1}{(N-k+2) !} \epsilon_{\alpha \beta \ldots \gamma \delta \ldots \rho} \underbrace{e^{\delta} \wedge \cdots \wedge e^{\rho}}_{N+2-k},
$$

and we denote the curvature of $\Gamma_{\beta}^{\alpha}$

$$
F^{\alpha \beta}:=\mathrm{d} \Gamma^{\alpha \beta}+\Gamma_{\gamma}^{\alpha} \wedge \Gamma^{\gamma \beta}
$$

The Palatini action for the vacuum Einstein equations in arbitrary dimension can be written as

$$
S=C \int_{M} F^{\alpha \beta} \wedge \Sigma_{\alpha \beta}+S_{\partial}
$$

with $C$ being a constant of dimension $L^{N}$. The boundary term $S_{\partial}$ is unnecessary in the properly quasi-local case

$$
S_{\partial}=0
$$

whereas in the asymptotically flat case we take it to be

$$
S_{\partial}=-C \lim _{r \rightarrow \infty} \int_{\tau_{r}} \Gamma^{\alpha \beta} \wedge \Sigma_{\alpha \beta}
$$

where $\tau_{r}$ stands for the cylinder (a sphere worldsheet) $r=$ const (see footnote 6). That additional boundary term is added to ensure that the variational problem with asymptotically flat boundary conditions is equivalent to the vacuum Einstein equations. Its role can be seen easily if we compute the variation of $S$ corresponding to an arbitrary vector field $\delta$ tangent to the phase space $\Gamma$, namely

$$
\delta S=\int_{M} S_{\Sigma_{\mu \nu}} \delta \Sigma_{\mu \nu}+\int_{M} S_{\Gamma_{\mu \nu}} \delta \Gamma_{\mu \nu}-C \lim _{r \rightarrow \infty} \int_{\tau_{r}} \Gamma^{\alpha \beta} \wedge \delta \Sigma_{\alpha \beta} .
$$

Now, it follows from the asymptotic flatness that the boundary term is zero, whereas the vanishing of the bulk terms is equivalent to the Einstein equations.

It is easy to check that in neither case do we need to add any surface term associated with $\Delta$. This is a consequence of the properties of non-expanding horizons [2, 5].

The covariant phase space is equipped with a pre-symplectic structure, i.e. an antisymmetric 2-form $\Omega$. It is defined by a pre-symplectic current calculated in the usual way: take two vector fields $\delta_{1}$ and $\delta_{2}$ tangent to $\Gamma$, and consider the following identity:

$$
\delta_{1} \delta_{2} S-\delta_{2} \delta_{1} S-\left[\delta_{1}, \delta_{2}\right] S=0 .
$$

If the Einstein equations are satisfied, the left-hand side takes the following form:

$\delta_{1} \delta_{2} S-\delta_{2} \delta_{1} S-\left[\delta_{1}, \delta_{2}\right] S=-C \int_{\partial M} \delta_{1} \Gamma^{\alpha \beta} \wedge \delta_{2} \Sigma_{\alpha \beta}-\delta_{2} \Gamma^{\alpha \beta} \wedge \delta_{1} \Sigma_{\alpha \beta}$

The boundary of $M$ consists of the two spacelike hyper-surfaces $M_{0}$ and $M_{1}$, the horizon $\Delta$, plus, in the asymptotically flat case, the cylinder $\tau_{r}$ where $r \rightarrow \infty$.

The differential $(N+1)$-form equal to $C$ times the integrant is called the pre-symplectic current

$$
j\left(\delta_{1}, \delta_{2}\right)=-C\left(\delta_{1} \Gamma^{\alpha \beta} \wedge \delta_{2} \Sigma_{\alpha \beta}-\delta_{2} \Gamma^{\alpha \beta} \wedge \delta_{1} \Sigma_{\alpha \beta}\right) .
$$

The pre-symplectic form may be defined by integrating the pre-symplectic current

$$
\boldsymbol{\Omega}\left(\delta_{1}, \delta_{2}\right)=\int_{\Delta_{0, v_{0}} \cup M_{v_{0}}} j\left(\delta_{1}, \delta_{2}\right)
$$




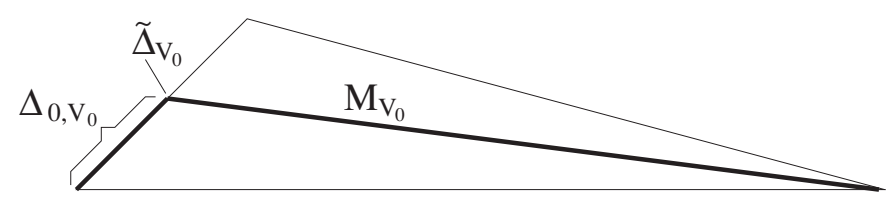

Figure 4. The integration surface for the symplectic form.

along any of the surfaces $\Delta_{0, v_{0}} \cup M_{v_{0}}$ (see figure 4) where $\Delta_{0, v_{0}}$ is the portion of the surface $\Delta$ bounded by the slices $\tilde{\Delta}_{0}$ such that $v=0$, and $\tilde{\Delta}_{v_{0}}$ such that $v=v_{0}$,

$$
\Delta_{0, v_{0}}:=\hat{\Delta} \times\left[0, v_{0}\right] .
$$

$M_{v_{0}}$ is an $(N+1)$-dimensional sub-manifold of $M^{\prime}$ whose boundary consists of $\tilde{\Delta}_{v_{0}}$ and: either $S$ in the quasi-local case or the 'sphere at infinity' in the asymptotically flat case with $M_{v_{0}}$ being asymptotically flat in the asymptotically flat coordinates (see footnote 6). Due to identity (38), the integral (40) is independent of the choice of surface labelled by any $0 \leqslant v_{0} \leqslant 1$. Also the domains of dependence of each of the surfaces which can be used in (40) are equal to the domain of dependence of $M_{0}$. In particular, we could choose $v_{0}=0$ and just $M_{0}$ as the integration surface. Therefore, the integral depends only on the definition of region $M$ and, in particular, on the choice of surface $M_{0}$ itself. Note that by considering an arbitrary value of $v_{0}$ in (40) we will be able to see explicitly what that dependence is like, and why our final results are independent of that choice.

In the integral defining the pre-symplectic form (40) the orientation of the surface $\Delta_{0, v_{0}} \cup M_{v_{0}}$ is the one given by the Stokes theorem applied to the region bounded by $M_{0}$ and $\Delta_{0, v_{0}} \cup M_{v_{0}}$. In particular, the volume forms on $\Delta_{0, v_{0}}$ and, respectively, $M_{v_{0}}$ are: $-\mathrm{d} v \wedge e^{1} \wedge \cdots \wedge e^{N}$, and $e^{N+2} \wedge e^{1} \wedge \cdots \wedge e^{N}$.

We will analyse the horizon part of the symplectic form first. The boundary conditions (26), (27), (28), (29), (30) imply that, in particular, given a gravitation field, the rotation 1 -form potential $\omega$ of the WIH $(\Delta,[l])$ defined by the vector field $l$ is given by the following pullback to the horizon:

$$
\left(\Gamma^{N+1 N+2}\right)_{\Delta}=-\omega .
$$

The induced null surface geometry of $\Delta$ determines the area $N$-form $\epsilon$ which can be expressed as

$$
\left(\Sigma_{N+1 N+2}\right)_{\Delta}=\left(e^{1} \wedge \cdots \wedge e^{N}\right)_{\Delta}=:-\epsilon .
$$

The area of arbitrary spacelike $N$-dimensional hyper-surface $\tilde{S} \subset \Delta$ is equal to $\int_{\tilde{S}} \epsilon$ (provided that the orientation of $\tilde{S}$ is fixed appropriately). On the other hand,

$$
\begin{aligned}
& \Sigma_{N+1 A}= \pm \underbrace{e^{1} \wedge \cdots \wedge e^{N}}_{\text {no } \mathrm{A}} \wedge e^{N+2} \\
& \Sigma_{N+2 A}= \pm \underbrace{e^{1} \wedge \cdots \wedge e^{N}}_{\text {no A }} \wedge e^{N+1} \\
& \Sigma_{A B}= \pm \underbrace{e^{1} \wedge \cdots \wedge e^{N}}_{\text {no A and B }} \wedge e^{N+1} \wedge e^{N+2} .
\end{aligned}
$$

Note that since $\Sigma_{N+1 A}$ and $\Sigma_{A B}$ contain $e^{N+2}$, they must vanish when pulled back to $\Delta$. We are left therefore with

$$
\int_{\Delta_{0, v_{0}}} j\left(\delta_{1}, \delta_{2}\right)=-2 C \int_{\Delta_{0, v_{0}}} \delta_{1} \omega \wedge \delta_{2} \epsilon-\delta_{2} \omega \wedge \delta_{1} \epsilon .
$$


We can simplify this expression if we decompose $\omega$ to

$$
\omega=\kappa^{(l)} \mathrm{d} v+\tilde{\omega}
$$

with $l\lrcorner \tilde{\omega}=0$. It is straightforward to prove that $\tilde{\omega} \wedge \epsilon=0$ and therefore the expression for the horizon part of the symplectic form can be further simplified as

$$
\int_{\Delta_{0, v_{0}}} j\left(\delta_{1}, \delta_{2}\right)=-2 C\left(\delta_{1} \kappa^{(l)} \int_{\Delta_{0, v_{0}}} \mathrm{~d}\left(v \delta_{2} \epsilon\right)-\delta_{2} \kappa^{(l)} \int_{\Delta_{0, v_{0}}} \mathrm{~d}\left(v \delta_{1} \epsilon\right)\right) .
$$

The horizon part of the identity can finally be integrated out to

$$
\int_{\Delta_{0, v_{0}}} j\left(\delta_{1}, \delta_{2}\right)=-2 v_{0} C\left(\delta_{1} \kappa^{(l)} \delta_{2} a_{\Delta}-\delta_{2} \kappa^{(l)} \delta_{1} a_{\Delta}\right)
$$

where $a_{\Delta}$ stands for the area of a cross-section of $\Delta \rightarrow \hat{\Delta}$.

We conclude that the symplectic form reads

$$
\begin{aligned}
\boldsymbol{\Omega}\left(\delta_{1}, \delta_{2}\right) & =-2 C v_{0}\left(\delta_{1} \kappa^{(l)} \delta_{2} a_{\Delta}-\delta_{2} \kappa^{(l)} \delta_{1} a_{\Delta}\right)-C \int_{M_{v_{0}}}\left(\delta_{1} \Gamma^{\alpha \beta} \wedge \delta_{2} \Sigma_{\alpha \beta}-\delta_{2} \Gamma^{\alpha \beta} \wedge \delta_{1} \Sigma_{\alpha \beta}\right) \\
& :=\Omega_{\Delta_{0, v_{0}}}\left(\delta_{1}, \delta_{2}\right)+\Omega_{\text {bulk }}\left(\delta_{1}, \delta_{2}\right)
\end{aligned}
$$

where $\Omega_{\text {bulk }}$ (the 'bulk' part) is the integral and the remaining (first) term constitutes $\boldsymbol{\Omega}_{\Delta_{0, v_{0}}}$ (the 'horizon' part). The horizon part is proportional to $v_{0}$, the distance in terms of the affine parameter corresponding to $l$, between the slices $\tilde{\Delta}_{v_{0}}$ and $\tilde{\Delta}_{0}$. The horizon part is invariant with respect to rescalings of $l$ by a constant, but it depends on the choice of the initial slice $\tilde{\Delta}_{0}$.

\section{Generating functions for spacetime diffeomorphisms}

A special class of vector fields tangent to the phase space $\Gamma$ is defined by the diffeomorphisms of $M$. Assign a vector field $X$ defined in a neighbourhood of $M$ to every point in $\Gamma$ (a solution of the Einstein equations on $M$ ). From our point of view, the flow of $X$ transports the gravitational field, while the region $M$ is kept fixed. A necessary condition for $X$ is that the flow of $X$ understood in this way preserves the boundary conditions (26), (27), (28), (29), (30) at $\Delta$.

The Lie derivative along $X$ defines a vector field $\delta_{X}$ tangent to $\Gamma$ (or a variation, as this object is often called in variational calculus). Our goal is to formulate conditions upon which the flow of $\delta_{X}$ is generated by a Hamiltonian function. Namely, we want to determine the conditions under which there exists a function $H$ on $\Gamma$ such that

$$
-\delta H=\Omega\left(\delta_{X}, \delta\right)
$$

for every vector field $\delta$ (variation) tangent to $\Gamma$ (in other words $\left.-\mathrm{d} H=\delta_{X}\right\lrcorner \Omega$, where all the operations apply to differential forms and vectors in $\Gamma$.)

Before we proceed, we introduce several formulae we will use in subsequent calculations.

We assume the vacuum Einstein equations to be satisfied on entire spacetime

$$
\begin{aligned}
& D \Sigma_{\alpha \beta} \equiv \mathrm{d} \Sigma_{\alpha \beta}-\Gamma_{\alpha}^{\gamma} \wedge \Sigma_{\gamma \beta}-\Gamma_{\beta}^{\gamma} \wedge \Sigma_{\alpha \gamma}=0 \\
& F^{\alpha \beta} \wedge \Sigma_{\alpha \beta \gamma}=0 .
\end{aligned}
$$

Those imply the following identities for the variations

$$
\begin{aligned}
& D \delta \Sigma_{\alpha \beta}=\delta \Gamma_{\alpha}^{\gamma} \wedge \Sigma_{\gamma \beta}-\delta \Gamma_{\beta}^{\delta} \wedge \Sigma_{\delta \alpha} \\
& \delta F^{\alpha \beta} \wedge \Sigma_{\alpha \beta \gamma}=-F^{\alpha \beta} \wedge \delta e^{\delta} \wedge \Sigma_{\alpha \beta \gamma \delta} .
\end{aligned}
$$


These are in fact the linearized Einstein equations.

Two useful identities follow from (31):

$$
\delta \Sigma_{\alpha \beta \gamma}=\delta e^{\delta} \wedge \Sigma_{\alpha \beta \gamma \delta}
$$

and

$$
X\lrcorner \Sigma_{\alpha \beta}=X^{\gamma} \Sigma_{\alpha \beta \gamma}
$$

with $X^{\gamma}=e^{\gamma}(X)$.

We now calculate $\Omega_{\text {bulk }}\left(\delta_{X}, \delta\right)$. The variations of the fields are equal to the Lie derivative along $X$. By applying the well-known Cartan formula $\left.\left.\mathcal{L}_{X} \alpha=\mathrm{d}(X\lrcorner \alpha\right)+X\right\lrcorner \mathrm{d} \alpha$ we get

$$
\begin{aligned}
\Omega_{\text {bulk }}\left(\delta_{X}, \delta\right)= & \left.-C \int_{M_{v_{0}}}\left(\mathrm{~d}(X\lrcorner \Gamma^{\alpha \beta}\right) \wedge \delta \Sigma_{\alpha \beta}+(X\lrcorner \mathrm{d} \Gamma^{\alpha \beta}\right) \wedge \delta \Sigma_{\alpha \beta} \\
& \left.\left.\left.-\delta \Gamma^{\alpha \beta} \wedge \mathrm{d}(X\lrcorner \Sigma_{\alpha \beta}\right)-\delta \Gamma^{\alpha \beta} \wedge(X\lrcorner \mathrm{d} \Sigma_{\alpha \beta}\right)\right) .
\end{aligned}
$$

This formula can be reduced to mere boundary terms. First we apply integration by parts to the first and third terms, the definition of curvature 2-form (32) to the second and (53) to the fourth. We get

$$
\begin{aligned}
\Omega_{\text {bulk }}\left(\delta_{X}, \delta\right)= & \left.\left.-C \int_{\partial M_{v_{0}}}\left((X\lrcorner \Gamma^{\alpha \beta}\right) \delta \Sigma_{\alpha \beta}+\delta \Gamma^{\alpha \beta} \wedge(X\lrcorner \Sigma_{\alpha \beta}\right)\right) \\
& \left.\left.-C \int_{M_{v_{0}}}\left(-\delta F^{\alpha \beta} \wedge(X\lrcorner \Sigma_{\alpha \beta}\right)+(X\lrcorner F^{\alpha \beta}\right) \wedge \delta \Sigma_{\alpha \beta}\right) .
\end{aligned}
$$

The remaining bulk term can be proved to vanish. By virtue of (58) and (56) we have

$$
\left.-\delta F^{\alpha \beta} \wedge(X\lrcorner \Sigma_{\alpha \beta}\right)=-X^{\gamma} \delta F^{\alpha \beta} \wedge \Sigma_{\alpha \beta \gamma}=X^{\gamma} F^{\alpha \beta} \wedge \delta e^{\delta} \wedge \Sigma_{\alpha \beta \gamma \delta} .
$$

The bulk term can now be rewritten in the form of

$$
\left.\left.\left.-\delta F^{\alpha \beta} \wedge(X\lrcorner \Sigma_{\alpha \beta}\right)+(X\lrcorner F^{\alpha \beta}\right) \wedge \delta \Sigma_{\alpha \beta}=\delta e^{\delta} \wedge\left(X^{\gamma} F^{\alpha \beta} \wedge \Sigma_{\alpha \beta \gamma \delta}-(X\lrcorner F^{\alpha \beta}\right) \wedge \Sigma_{\alpha \beta \delta}\right) .
$$

The last term is just the contraction of the right-hand side of Einstein equations (56) with $X$ and is therefore equal to $0 .^{8}$ We are left only with the surface terms of the bulk part of the symplectic form

$$
\left.\left.\Omega_{\text {bulk }}\left(\delta_{X}, \delta\right)=-C \int_{\partial M_{v_{0}}}\left((X\lrcorner \Gamma^{\alpha \beta}\right) \delta \Sigma_{\alpha \beta}+\delta \Gamma^{\alpha \beta} \wedge(X\lrcorner \Sigma_{\alpha \beta}\right)\right) .
$$

The horizon part of the symplectic form, on the other hand, vanishes,

$$
\Omega_{\Delta_{0, v_{0}}}\left(\delta_{X}, \delta\right)=-2 C v_{0}\left(\mathcal{L}_{X} \kappa^{(l)} \delta a_{\Delta}-\delta \kappa^{(l)} \mathcal{L}_{X} a_{\Delta}\right)=0,
$$

because both the area $a_{\Delta}$ and the surface gravity of $l$ are constant on a WIH.

Finally

$$
\left.\left.\boldsymbol{\Omega}\left(\delta_{X}, \delta\right)=-C \int_{\partial M_{v_{0}}}\left((X\lrcorner \Gamma^{\alpha \beta}\right) \delta \Sigma_{\alpha \beta}+\delta \Gamma^{\alpha \beta} \wedge(X\lrcorner \Sigma_{\alpha \beta}\right)\right) .
$$

Every vector field $X$ can be split into two parts, say $X^{\prime}$ and $X^{\prime \prime}$, one being identically zero outside some finite neighbourhood of the horizon while the other identically vanishes at the horizon. In the asymptotically flat case, the contribution of $X^{\prime \prime}$ to (65) is the variation of some

8 All calculations shown here do not differ significantly from the four-dimensional case. This may be traced back to the fact that in all wedge products of forms the first forms are either the curvature 2-form $F^{\alpha \beta}$ or the connection 1 -form $\Gamma^{\alpha \beta}$. 
ADM momentum (see [5]), provided that $X^{\prime \prime}$ satisfies appropriate conditions in the infinity. $X^{\prime}$, on the other hand, is responsible for the horizon contribution to (65).

Our goal is to derive the contribution from the horizon. We assume $X$ is identically zero out of a finite neighbourhood of the horizon, and in the properly quasi-local case we assume the support $\operatorname{supp}(X)$ intersects the boundary of $M_{v_{0}}$ at $\Delta$ only,

$$
\operatorname{supp}(X) \cap \delta M_{v_{0}} \subset \Delta .
$$

Suppose that $X$ satisfies the following boundary condition on the horizon:

$$
\left.X\right|_{\Delta}=\frac{\kappa^{(X)}}{\kappa^{(l)}} l-\Omega^{(X)} \phi,
$$

where the quantities $\kappa^{(X)}$ and $\Omega^{(X)}$ are constant on the horizon, but their values possibly depend on the gravitational field ${ }^{9}$. That is, $\kappa^{(X)}$ and $\Omega^{(X)}$ are functions defined on the phase space $\Gamma$. (One should not confuse $\Omega^{(X)}$ with the symplectic form.) The reason for this notation as well as the geometric and physical meaning of the two quantities introduced here will be explained in the next three sections. Form (67) of $X$ at $\Delta$ is a natural assumption in the case of a WIH admitting a non-null symmetry generator (compare with (22)). We discuss this case in section 8 .

By substituting $X$ into (65) by the right-hand side of (67) and taking into account the boundary conditions at $\Delta((26),(27)$ and $(28))$ we get $^{10}$

$$
\begin{aligned}
\Omega\left(\delta_{X}, \delta\right) & \left.\left.=-2 C \int_{\tilde{\Delta}_{v_{0}}}((X\lrcorner \omega) \delta \epsilon+\delta \omega \wedge(X\lrcorner \epsilon\right)\right) \\
& \left.\left.=-2 C \int_{\tilde{\Delta}_{v_{0}}}\left(\kappa^{(X)} \delta \epsilon-\Omega^{(X)}(\phi\lrcorner \omega\right) \delta \epsilon-\Omega^{(X)}(\phi\lrcorner \delta \omega\right) \epsilon\right) \\
& \left.=-2 C \int_{\tilde{\Delta}_{v_{0}}}\left(\kappa^{(X)} \delta \epsilon-\Omega^{(X)} \delta((\phi\lrcorner \omega) \epsilon\right)\right) \\
& \left.=2 C \kappa^{(X)} \delta a_{\Delta}+2 C \Omega^{(X)} \delta\left(\int_{\tilde{\Delta}_{v_{0}}}(\phi\lrcorner \omega\right) \epsilon\right) .
\end{aligned}
$$

The sign of the first term of the result follows from the orientation of $\tilde{\Delta}_{v_{0}}$ defined by the Stokes theorem, in which $\epsilon$ is minus the $N$-volume element.

\section{Angular momentum}

We now assume that the vector field $X$ coincides with the fixed vector field $\phi$ on the horizon, i.e. $\Omega^{(X)}=-1$ and $\kappa^{(X)}=0$. Then, $\Omega\left(\delta_{X}, \delta\right)$ calculated in (68) is necessarily a variation

$$
\left.\Omega\left(\delta_{\phi}, \delta\right)=-2 C \int_{\tilde{\Delta}_{v_{0}}} \delta(\phi\lrcorner \omega\right) \epsilon=:-\delta J^{(\phi)},
$$

where the generator

$$
\left.J^{(\phi)}:=2 C \int_{\tilde{\Delta}_{v_{0}}}(\phi\lrcorner \omega\right) \epsilon
$$

will be called the WIH angular momentum associated with the vector field $\phi$ tangent to $\Delta$. (We recall that the orientation of $\tilde{\Delta}_{v_{0}}$ is such that $\epsilon$ is minus the area form.) Due to the fact

$9 X$ is well defined for the gravitational fields such that $\kappa^{(l)} \neq 0$. In the four (spacetime) dimensional case the resulting Hamiltonian may be extended by continuity to the extremal points of $\boldsymbol{\Gamma}$ as well.

${ }^{10}$ We use below the following identity satisfied by the pullbacks of the involved differential forms onto $\tilde{\Delta}_{v_{0}}$ : $(\omega \wedge \epsilon)_{\tilde{\Delta}_{v_{0}}}=0$. 
that $\phi\lrcorner \omega$ is a function constant along the null geodesic generators of $\Delta$ (for both $\phi$ and $\omega$ are Lie dragged by $l$ ), and the area $N$-form is both Lie dragged by and orthogonal to $l$, the horizon angular momentum $J^{(\phi)}$ is independent of $v_{0}$. The formula agrees with the one given in [5] in the case of four-dimensional horizons.

\section{Area as a generator of null translations}

If we take $X$ to be just a null vector proportional to $l$ at $(\Delta,[l])$, i.e. $\Omega^{(X)}=0, \kappa^{(X)} \neq 0$, we get from (68)

$$
-\delta H_{X}=2 C \kappa^{(X)} \delta a_{\Delta} .
$$

The necessary condition for the flow to be Hamiltonian is that $H_{X}$ and $\kappa^{(X)}$ be functions of $a_{\Delta}$ solely. Conversely, given any function $H$ of the area, one can construct the corresponding Hamiltonian vector field $\delta_{X}$ in the following way. Fix a vector field $Y$ in $M$, such that $\left.Y\right|_{\Delta}=l$, and $Y$ vanishes out of a sufficiently small neighbourhood of $\Delta$. Given a gravitational field, i.e. a point in $\Gamma$, define in $M$ a vector field $X$,

$$
X:=-\frac{1}{2 C_{\kappa}^{(l)}} H^{\prime} Y,
$$

where $H^{\prime}$ is the derivative of $H$. Then, the corresponding vector field $\delta_{X}$ tangent to $\Gamma$ satisfies

$$
-\delta H=\Omega\left(\delta_{X}, \delta\right)
$$

for every vector field $\delta$ tangent to $\Gamma$.

In particular, the horizon area itself is a generator of a semigroup of diffeomorphisms, whose flow of the horizon $\Delta$ is defined by the null vector field $\left(-2 C \kappa^{(l)}\right)^{-1} l$. In other words, the horizon area is a generator of the null translations of the horizon, as was first observed in the case of the Killing horizons in 4-spacetime dimensions in [13] and shown for WIHs in four spacetime dimensions in [5].

\section{The first law}

In this section we will show that the requirement that the evolution of the gravitational field be Hamiltonian for general vector fields $X$ satisfying (67) implies a first law of black-hole thermodynamics analogous to the case of four spacetime dimensions.

Assume the left-hand side of (68) is a total variation of a quantity $H_{\Delta}$. The equality reads

$$
-\delta\left(H_{\Delta}\right)=2 C \kappa^{(X)} \delta a_{\Delta}+\Omega^{(X)} \delta J^{(\phi)} .
$$

Necessary conditions are:

- $H_{\Delta}, \kappa^{(X)}$ and $\Omega^{(X)}$ are arbitrary functions of $a_{\Delta}$ and $J^{(\phi)}$ only,

- an integrability condition analogous to the Maxwell relation in thermodynamics holds:

$$
2 C \frac{\partial \kappa^{(X)}}{\partial J^{(\phi)}}=\frac{\partial \Omega^{(X)}}{\partial a_{\Delta}} .
$$

Equation (52) imposes therefore strong constraints on the coefficients of the vector field $X$ at $\Delta$.

The dependence of $H_{\Delta}$ on $a_{\Delta}$ and $J^{(\phi)}$ is arbitrary. In the four-spacetime-dimensional case some additional conditions on the vector field $X$ were imposed [5], which eventually determined the generating function $H_{\Delta}$ completely. Firstly, it was not assumed that $X$ was zero except for a neighbourhood of $\Delta$. Instead, it was assumed that at the spatial infinity $X$ was 
a normalized generator of a time translation. Then, the corresponding function $H_{X}$ consists of two contributions, one from $\Delta$ and another from infinity, namely

$$
H_{X}=H_{\mathrm{ADM}}-H_{\Delta},
$$

where $H_{\mathrm{ADM}}$ is the ADM mass. Secondly, it was assumed that $X$ was assigned to each gravitational field, point in our phase space $\Gamma$, in such a way that, whenever the spacetime was asymptotically flat and stationary, $X$ coincided with the Killing vector field. If this assumption can be satisfied, then

$$
\delta_{X}=0, \quad \Omega\left(\delta_{X}, \delta\right)=0,
$$

and, in consequence

$$
\delta\left(H_{\Delta}\right)=\delta\left(H_{\mathrm{ADM}}\right)
$$

for every stationary gravitational field. The two equalities determine $H_{\Delta}$ by the ADM mass of the Kerr solution, modulo a constant. This is possible due to the uniqueness of a stationary, asymptotically flat, vacuum black hole of a given area $a_{\Delta}$ and angular momentum $J^{(\phi)}$. Since there is no natural constant of the right units in the vacuum case, the undetermined constant was set to zero. Remarkably, the resulting $H_{\Delta}$ extends smoothly to the extremal WIH case. (Incidently, it was not proven that a suitable assignment of $X$ to every point $\Gamma$ really exists. The conditions used were necessary only.)

As we mentioned in the introduction, the uniqueness no longer holds for asymptotically flat, axially symmetric vacuum spacetimes in the general, $(N+2)$-dimensional case. The issue is similar to that of hairy black holes [14] in four dimensions.

However, if one restricts the phase space $\Gamma$ to spherically symmetric (static) gravitational fields [6-8], then, just as in the four-dimensional case, the value of energy $H_{\Delta}$ as a function of horizon area can be determined. It is given by the function describing the ADM mass as a function of the horizon area (see [9])

$$
H_{\Delta}=C N\left(\frac{2 \pi^{\frac{N+1}{2}}}{\Gamma\left(\frac{N+1}{2}\right)}\right)^{\frac{1}{N}} A^{1-\frac{1}{N}}
$$

with $\Gamma(x)$ denoting Euler's gamma function.

\section{Invariance and non-invariance}

Let us discuss now the gauge invariance of our framework. In order to define the angular momentum and derive first law (74) we have equipped a non-expanding horizon $\Delta$ with extra structure: a WIH flow $[l]$, a foliation, a representative $l \in[l]$ and a vector field $\hat{\phi}$ defined on the space $\Delta$ of the null generators of $\Delta$. Also, the transversal surface $M_{0}$ was used for the definition of the pre-symplectic form. It is easy to observe that the generator of the null translations of $\Delta$, the area functional $a_{\Delta}$ is in fact uniquely defined given a non-expanding horizon only, and it is determined just by any cross-section. The definition of the second relevant parameter, the angular momentum $J^{(\phi)}$, involves all the elements of extra structure. We show below, however, that if the vector field $\hat{\phi}$ generates a local symmetry of the area element induced in $\hat{\Delta}$, then a corresponding $J^{(\phi)}$ depends only on the geometry of $\Delta$ and on the vector field $\hat{\phi}$ itself. And again it is determined by an arbitrary cross-section of $\Delta$. Indeed, integral (70) can be written as an integral along the base manifold $\hat{\Delta}$, namely

$$
\left.J^{(\phi)}=-2 C \int_{\hat{\Delta}}(\hat{\phi}\lrcorner \hat{\omega}\right) \hat{\epsilon}
$$


where $\hat{\epsilon}$ is the area element defined on $\hat{\Delta}$ by the induced metric tensor $\hat{q}$ and $\hat{\omega}$ is the pullback of the rotation 1 -form potential $\omega$ by the map

$$
\hat{\Delta} \rightarrow \tilde{\Delta}_{v_{0}}
$$

naturally defined, given a cross-section $\tilde{\Delta}_{v_{0}}$. Now, given a non-expanding horizon geometry on $\Delta$, a change of the WIH flow $[l]$ and the foliation, both used to define $\hat{\omega}$, amounts to a transformation

$$
\hat{\omega} \mapsto \hat{\omega}+\mathrm{d} g, \quad \text { where } \quad g: \hat{\Delta} \rightarrow \mathbb{R} .
$$

Consequently

$$
\begin{aligned}
\left.\int_{\hat{\Delta}}(\hat{\phi}\lrcorner \hat{\omega}^{\prime}\right) \hat{\epsilon} & \left.=\int_{\hat{\Delta}} \hat{\omega}^{\prime} \wedge(\hat{\phi}\lrcorner \hat{\epsilon}\right) \\
& \left.=\int_{\hat{\Delta}}(\hat{\omega}+\mathrm{d} g) \wedge(\hat{\phi}\lrcorner \hat{\epsilon}\right) \\
& \left.=\int_{\hat{\Delta}}(\hat{\omega} \wedge(\hat{\phi}\lrcorner \hat{\epsilon})+g\left(\mathcal{L}_{\hat{\phi}} \hat{\epsilon}\right)\right) \\
& \left.=\int_{\hat{\Delta}}(\hat{\phi}\lrcorner \hat{\omega}\right) \hat{\epsilon}+\int_{\hat{\Delta}} g \mathcal{L}_{\hat{\phi}} \hat{\epsilon} .
\end{aligned}
$$

Therefore, in the most elegant formulation of this framework one can add one more boundary condition, namely the assumption that for every gravitational field in $\Gamma$ the WIH defined on the surface $\Delta$ by the vector field $l$ admits a non-null symmetry generator $Y$ such that $\Pi_{*} Y$ is a fixed vector field $\hat{\phi}$ on $\hat{\Delta}$. A generic WIH of this type admits exactly a two-dimensional group of symmetry generators 2.4. Then, our assumption (67) is equivalent to assuming that the vector field $X$ restricted to $\Delta$ is a symmetry generator.

\section{Discussion and conclusions}

We proved that the first law of black-hole thermodynamics holds for arbitrarily dimensional weakly isolated horizons. We have defined the horizon angular momentum as the generator of rotational symmetry. We have shown that the assumption that time flow on the horizon is Hamiltonian leads to a differential condition on the mass function which can be interpreted as the first law of thermodynamics of black holes.

We also proposed a Hamiltonian function depending on the horizon area and angular momentum.

\section{Acknowledgments}

We would like to thank Abhay Ashtekar and Jerzy Kijowski for discussions. This work was supported in part by the Polish Committee for Scientific Research (KBN) under grants no 2 P03B 127 24, 2 P03B 130 24, the National Science Foundation under grant 0090091, and the Albert Einstein Institute of the Max Planck Society.

\section{References}

[1] Lewandowski J and Pawłowski T 2005 Geometry of non-expanding horizons: $n$-dimensional generalization Class. Quantum Grav. 22 1573-98 (Preprint gr-qc/0410146)

[2] Ashtekar A, Fairhurst S and Krishnan B 2000 Isolated horizons: Hamiltonian evolution and the first law Phys. Rev. D 62104025 (Preprint gr-qc/0005083) 
[3] Ashtekar A, Beetle C, Dreyer O, Fairhurst S, Krishnan B, Lewandowski J and Wiśniewski J 2000 Generic isolated horizons and their applications Phys. Rev. Lett. $853564-7$ (Preprint gr-qc/0006006)

[4] Ashtekar A, Beetle C and Lewandowski J 2002 Geometry of generic isolated horizons Class. Quantum Grav. 19 1195-225 (Preprint gr-qc/0111067)

[5] Ashtekar A, Beetle C and Lewandowski J 2001 Mechanics of rotating isolated horizons Phys. Rev. D 64 044016 (Preprint gr-qc/0103026)

[6] Rogatko M 2002 Uniqueness theorem for static black hole solution of $\sigma$-models in higher dimensions Class. Quantum Grav. 19 L151 (Preprint hep-th/0207187)

[7] Rogatko M 2003 Uniqueness theorem of static degenerate and non-degenerate charged black holes in higher dimensions Phys. Rev. D 67084025 (Preprint gr-qc/0302091)

[8] Rogatko M 2004 Uniqueness theorem for generalized Maxwell electric and magnetic black holes in higher dimensions Phys. Rev. D 70044023 (Preprint gr-qc/0406041)

[9] Myers R C and Perry M J 1986 Ann. Phys. 172304

[10] Emparan R and Reall H S 2002 A rotating black ring in five dimensions Phys. Rev. Lett. 88 101101 (Preprint hep-th/0110260)

[11] Morisawa Y and Daisuke I 2004 A boundary value problem for the five-dimensional stationary rotating black holes Phys. Rev. D 69124005 (Preprint gr-qc/0401100)

[12] Czuchry E, Jezierski J and Kijowski J 2004 Local approach to thermodynamics of black holes Relativity Today (Proc. 7th Hungarian Relativity Workshop 2003) ed I Racz (Budapest: Akadémiai Kiadó) (Preprint gr-qc/0405073)

[13] Wald B 1993 Black hole entropy is the Noether charge Phys. Rev. D 48 R3427-31

[14] Ashtekar A, Corichi A and Sudarsky D 2001 Hairy black holes, horizon mass and solitons Class. Quantum Grav. 18 919-40

[15] Ashtekar A, Baez J and Krasnov K 2000 Quantum geometry of isolated horizons and black hole entropy Adv. Theor. Math. Phys. 4 1-94 (Preprint gr-qc/0005126)

[16] Ashtekar A and Krishnan B 2004 Isolated and dynamical horizons and their applications Liv. Rev. Rel. 710 (Preprint gr-qc/0407042)

[17] Czuchry E, Jezierski J and Kijowski J 2004 Dynamics of gravitational field within a wave front and thermodynamics of black holes Phys. Rev. D 70124010 (Preprint gr-qc/0412042) 